\title{
Cardiovascular screening to reduce the burden from cardiovascular disease: microsimulation study to quantify policy options
}

\author{
Chris Kypridemos, ${ }^{1}$ Kirk Allen, ${ }^{1,2}$ Graeme L Hickey, ${ }^{3}$ Maria Guzman-Castillo, ${ }^{1}$ Piotr Bandosz, ${ }^{1,4}$ \\ lain Buchan, ${ }^{5}$ Simon Capewell, ${ }^{1}$ Martin O'Flaherty ${ }^{1}$
}

${ }^{1}$ Department of Public Health

and Policy, University of

Liverpool, Liverpool L69 3GB,

UK

2Lancaster Medical School,

Lancaster University, Lancaster,

UK

${ }^{3}$ Department of Biostatistics,

University of Liverpool,

Liverpool, UK

${ }^{4}$ Department of Prevention and

Medical Education, Medical

University of Gdansk, Gdansk,

Poland

5Farr Institute @ HeRC,

University of Manchester,

Manchester, UK

Correspondence to:

C Kypridemos

c.kypridemos@liverpool.ac.uk

Additional material is published online only. To view please visit

the journal online.

Cite this as: BMJ 2016;353:i2793

http://dx.doi.org/10.1136/bmj.i2793

Accepted: 09 May 2016

\section{ABSTRACT}

\section{OBJECTIVES}

To estimate the potential impact of universal screening for primary prevention of cardiovascular disease (National Health Service Health Checks) on disease burden and socioeconomic inequalities in health in England, and to compare universal screening with alternative feasible strategies.

\section{DESIGN}

Microsimulation study of a close-to-reality synthetic population. Five scenarios were considered: baseline scenario, assuming that current trends in risk factors will continue in the future; universal screening; screening concentrated only in the most deprived areas; structural population-wide intervention; and combination of population-wide intervention and concentrated screening.

\section{SETTING}

Synthetic population with similar characteristics to the community dwelling population of England.

\section{PARTICIPANTS}

Synthetic people with traits informed by the health survey for England.

\section{MAIN OUTCOME MEASURE}

Cardiovascular disease cases and deaths prevented or postponed by 2030 , stratified by fifths of socioeconomic status using the index of multiple deprivation.

RESULTS

Compared with the baseline scenario, universal screening may prevent or postpone approximately

\section{WHAT IS ALREADY KNOWN ON THIS TOPIC}

Two main strategies for the primary prevention of cardiovascular disease (CVD) is to screen the population, find those individuals at high risk, and treat them or to reduce the CVD risk of the whole population irrespective of individuals' baseline risk Evidence suggests that the second approach is more effective and likely more equitable, yet this depends on the distribution of CVD risk throughout the population In England, the Department of Health adopted the first approach, although this decision has recently attracted some criticism

\section{WHAT THIS STUDY ADDS}

In England, despite the observed higher concentration of CVD risk in more deprived areas, structural population-wide interventions targeting unhealthy diet and tobacco might be three times more effective than the existing screening policy Structural population-wide interventions are also likely to be more equitable than screening

A comprehensive strategy, combining structural population-wide interventions with screening in the most deprived areas (where CVD risk is concentrated) is most likely to maximise both effectiveness and equity of primary CVD prevention
19000 cases (interquartile range $11000-28000$ ) and 3000 deaths ( $-1000-6000)$; concentrated screening 17000 cases (9000-26000) and 2000 deaths (-1000-5000); population-wide intervention 67000 cases (57 000-77 000) and 8000 deaths (4000-11000); and the combination of the population-wide intervention and concentrated screening 82000 cases (73000-93000) and 9000 deaths (6000-13000). The most equitable strategy would be the combination of the population-wide intervention and concentrated screening, followed by concentrated screening alone and the population-wide intervention. Universal screening had the least apparent impact on socioeconomic inequalities in health.

\section{CONCLUSIONS}

When primary prevention strategies for reducing cardiovascular disease burden and inequalities are compared, universal screening seems less effective than alternative strategies, which incorporate population-wide approaches. Further research is needed to identify the best mix of population-wide and risk targeted CVD strategies to maximise cost effectiveness and minimise inequalities.

\section{Introduction}

Cardiovascular disease (CVD) is the leading cause of death worldwide. ${ }^{1}$ Furthermore, substantial socioeconomic inequalities have been observed in CVD mortality in England and elsewhere. ${ }^{23}$ These inequalities powerfully reflect much greater premature mortality, and hence shorter life expectancy, among the most deprived groups. In England, the current governmental action plan to tackle the burden of CVD includes a programme known as NHS (National Health Service) Health Checks. Introduced in 2009, this programme promotes the screening of all healthy adults aged 40 to 74 for CVD risk stratification, and treatment of those at high risk. ${ }^{45}$ Recently, the debate about the programme's scientific foundation, effectiveness, and cost effectiveness, however, has been heated. ${ }^{6-10}$ Despite the controversy, the programme remains policy.

Beyond the obvious importance of the debate to national public health, the programme's relevance extends internationally. Choices about public health policy in the United Kingdom influence policy worldwide; the UK policies on tobacco control and salt reduction are two recent examples. ${ }^{112}$ In essence, the debate about NHS Health Checks originates from the archetypal debate of targeted "high risk" versus "population-wide" preventive interventions that was first articulated by Geoffrey Rose. ${ }^{13}$ Rose argued that 
population-wide interventions are more effective than ones aimed at high risk groups because the majority of incident cases occur in the multitudinous group of people at low and intermediate risk. In Rose terminology, NHS Health Checks is a typical "high risk intervention," as it targets people at high risk rather than lowering risk in the whole population.

The effectiveness of high risk interventions for CVD prevention has been previously challenged. ${ }^{14}$ More recently, a Cochrane systematic review and the Inter99 trial found no benefits of health checks on CVD morbidity or mortality. ${ }^{15} 16$ There were, however, major limitations to these studies: Inter99 trialled a counselling intervention not supported by additional drug treatment, and in the Cochrane review nine out of 14 trials were conducted before 1980, when the treatment options for high risk people were limited. In addition, high risk interventions may be more effective in populations with high clustering of risk factors, resulting in a high concentration of the risk to certain groups in the population. ${ }^{17}$ In fact, the English population has such characteristics, with the risk of CVD being higher among those in the most socioeconomically deprived groups. ${ }^{18}$

High risk interventions may generate health inequalities because they require active participation of people in both screening and treatment of those at high risk, favouring those with more resources. ${ }^{1419-21}$ The particular effect of NHS Health Checks on socioeconomic health inequalities remains unclear however. A national study reported no difference in the coverage of the intervention by deprivation, ${ }^{22}$ whereas several smaller, but more detailed, studies showed substantially lower uptake in deprived areas. ${ }^{23-25}$

We estimated the potential impact of universal screening for primary prevention of CVD on disease burden and socioeconomic health inequalities in England. Available data on the effectiveness of the NHS Health Check programme have been used to model this scenario. We further compared universal CVD screening with an alternative approach targeting only deprived areas, a feasible population-wide intervention, and a combination of both.

\section{Methods}

Building on experience from the original, validated IMPACT model ${ }^{26}$ and the more recent IMPACT $_{\mathrm{SEC}^{27}}$ and IMPACT2 models, ${ }^{28}$ we created IMPACT $_{\mathrm{NCD}}$, a discrete time dynamic stochastic microsimulation model. IMPACT $_{\text {NCD }}$ simulates the life course of synthetic individuals under different counterfactual scenarios, up to 2030 (the projection horizon). During the simulation, CVD incidence and CVD and non-CVD mortality are recorded. The results are stratified by year, five year age group, sex, and fifths of index of multiple deprivation. The last is a relative measure of area deprivation that is widely used by public health authorities in England, and it has been used as the measure of socioeconomic classification for this study. ${ }^{29}$

A more detailed description of the model is provided in the supplementary material and the source code is available at https://github.com/ChristK/IMPACTncd/ tree/CVD-policy-options.

\section{Scenarios}

We considered five scenarios.

\section{Baseline (current trends)}

In the baseline scenario, we assumed that the recent observed trends in CVD risk factor trajectories by age, sex, and socioeconomic status will continue in the near future. We extracted the trends from the health survey for England 2001-12, a nationally representative series of health surveys conducted in England annually. ${ }^{30-42}$

\section{Universal screening}

This scenario modelled the potential health effects of universal screening to identify and treat people at high risk for CVD. Input variables were informed from current implementation of the NHS Health Check programme. Eligible people were defined as adults aged between 40 and 74, excluding those with a known history of CVD, atrial fibrillation, diabetes mellitus, rheumatoid arthritis, or renal disease; closely resembling real life eligibility criteria. Based on existing evidence we assumed an uptake of $50 \%$ for screening, ${ }^{43}$ and we calibrated the distribution of the estimated 10 year risk of developing CVD among those participating: $70 \%$ with a less than $10 \%$ risk, $25 \%$ with between $10 \%$ and $20 \%$, and $5 \%$ with more than $20 \%{ }^{22}$ In addition, we calibrated the age distribution so that around $30 \%$ of those screened were older than $60 .{ }^{22}$ Participants with a higher than $10 \%$ estimated 10 year risk of developing CVD were considered at high risk and eligible for treatment. We used the QRISK2 score to estimate the 10 year risk of developing CVD, as perceived from healthcare. ${ }^{4} 4$

Based on published evidence, we assumed that about $24 \%$ with an estimated risk of $20 \%$ or more and total cholesterol of $5 \mathrm{mmol} / \mathrm{L}$ or more will be prescribed atorvastatin $20 \mathrm{mg}$ and about $27 \%$ with an estimated risk of $20 \%$ or more and a systolic blood pressure of $135 \mathrm{~mm} \mathrm{Hg}$ or more will be prescribed antihypertensive drugs. For those with a risk between $10 \%$ and $20 \%$ we assumed that about $17 \%$ and $20 \%$ will be prescribed treatment, respectively. ${ }^{45}$ We assumed an $80 \%$ persistence with treatment and a mean adherence of approximately $70 \%$, roughly based on evidence from Denmark. ${ }^{46}$ Moreover, we modelled high risk participants with a body mass index of more than $50 \mathrm{~kg} / \mathrm{m}^{2}$ to undergo bariatric surgery and reduce their body mass index to $30 \mathrm{~kg} / \mathrm{m}^{2}$. We assumed that with lifestyle counselling half of the high risk participants consuming fewer than five fruit and vegetable portions daily will increase their consumption by a portion daily. Half of those being active for less than five days a week will increase their physical activity by an active day each week, and all high risk participants will decrease their body mass index by around $1 \% .{ }^{4547}$ Finally, we modelled $10 \%$ of high risk smokers to achieve cessation for a year and have a probability of relapse equal to that of the general population by sex, fifth of multiple deprivation, and years since cessation. 4849 


\section{Concentrated screening}

In the concentrated screening scenario, we simulated a hypothetical strategy where screening had only been implemented in the most deprived fifths (groups 4 and 5), the groups with the greatest concentration of CVD risk. We assumed that the uptake of the intervention was $50 \%$ and the risk and age distribution in the participants was similar to that in the eligible population. Otherwise, the strategy is similar to the previous universal screening scenario. Given the recent criticism about the cost and cost effectiveness of the intervention, ${ }^{9}$ offering the intervention where the risk is more concentrated may reduce costs.

\section{Population-wide intervention}

This scenario modelled the effects of a feasible population-wide structural intervention targeting unhealthy diet and smoking. Several studies have found that a tax on sugar sweetened beverages may reduce the prevalence of obesity. ${ }^{50-52}$ For this scenario we assumed that such a tax may reduce the mean increase in body mass index by about 5\% annually. Moreover, the United Kingdom has had one of the world's most successful salt reduction strategies, including public awareness campaigns, food labelling, and voluntary reformulation of processed foods..$^{53}$ Modelling studies suggested that the addition of mandatory reformulation of processed foods may further reduce mean systolic blood pressure by $0.8 \mathrm{~mm} \mathrm{Hg} ;{ }^{54}$ we modelled this decrease. A large randomised trial in the United States showed that subsidies on fruits and vegetables may increase consumption by about half a portion daily, and a modelling study in the UK found that subsidising fruits and vegetables combined with taxation of unhealthy foods may increase fruit and vegetable annual consumption by about $10 \% .{ }^{5556}$ We modelled an increase of a portion of fruit and vegetable each day in $50 \%$ of the population. Finally, a SimSmoke modelling study estimated that full compliance with the framework convention on tobacco control may reduce smoking prevalence by $13 \%$ (relative) in five years; ${ }^{57}$ we modelled this decrease.

\section{Population-wide intervention and concentrated screening}

This scenario is the combination of the population-wide intervention and concentrated screening strategies. We modelled the implementation of a population-wide strategy identical to the previous scenario, complemented by concentrated screening for people at high risk of CVD in the most deprived fifths (groups 4 and 5).

\section{Common scenario assumptions}

All interventions begun in 2011 and were linearly diffused into the population over a five year period. Trends in population risk factors were assumed to be the same as those of the baseline scenario for all but the population-wide intervention. All of the scenarios assumed that CVD case fatality will keep improving by $3 \%$ (relative) annually. In addition, we assumed a socioeconomic gradient in CVD case fatality, forcing the more deprived people to experience worse outcomes. Both case fatality assumptions were based on recent trends and are supported by the British Heart Foundation's statistics on coronary heart disease. ${ }^{2}$ Finally, a five year lag time was assumed between exposure to cardiovascular risk factors and disease.

\section{Model description}

Inputs and logic

IMPACT $_{\mathrm{NCD}}$ synthesises information from the Office for National Statistics and the health surveys for England on the English population's demographics and its exposure to CVD associated risk factors, to generate a close-to-reality synthetic population..$^{58}$ Well established causal pathways between CVD and the associated risk factors are used to translate exposure into CVD incidence and mortality, in a competing risk framework. We obtained effect sizes for exposures from published meta-analyses and longitudinal studies (see supplementary table S1).

The risk factors we considered for this study were age, sex, fifth of deprivation, body mass index, systolic blood pressure, total cholesterol level, diabetes mellitus (diagnosis or increased glycated haemoglobin level/no diabetes), smoking status (current, former, or never smoker), environmental tobacco exposure (binary variable), fruit and vegetable consumption (portions daily), and physical activity (days with at least 30 minutes of moderate or vigorous physical activity each week). CVD was defined as the sum of coronary heart disease and stroke (any type) cases. As this study focuses on primary prevention, we considered only the first ever episode of coronary heart disease or stroke. The competing risk framework allowed people to develop coronary heart disease and/or stroke separately, and to die from these two diseases or any other cause.

\section{Model outputs}

We report the cumulative estimates of cases and deaths prevented or postponed as measures of overall effectiveness of the modelled interventions. To measure the impact of the modelled interventions on absolute and relative socioeconomic health inequalities, we developed and used two regression based metrics inspired by the slope index of inequality; ${ }^{59}$ the absolute equity slope index and the relative equity slope index. The absolute equity slope index measures the impact of an intervention on absolute inequality; for example, a value of 100 means 100 more cases were prevented or postponed in most deprived areas compared with least deprived areas, resulting in a decrease in absolute inequality. The relative equity slope index takes into account the pre-existing socioeconomic gradient of disease burden and measures the impact of an intervention on relative inequality. Positive values mean the intervention tackles relative inequalities and negative values that the intervention generates relative inequality. Finally, we summarised the overall impact of each scenario on CVD burden and equity in the equity summary chart. 


\section{Uncertainty and sensitivity analysis}

IMPACT $_{\text {NCD }}$ implements a second order Monte Carlo design that allows uncertainty to be quantified from the outputs. We used distributions to model the uncertainty around all scenario specific inputs and the sampling error of the risk associated with the CVD related risk factors. The probabilistic sensitivity analysis has been incorporated in our estimates. We summarise the distributions by reporting medians and interquartile ranges in the form of first and third fourths. The supplementary file provides a more detailed description of the sources of uncertainty and the relevant distributions.

We ran three further scenarios offering slight variations on the two primary ones of universal screening and population-wide intervention: a universal screening variation, where we assumed a treatment threshold recommendation of $20 \%$ risk instead of $10 \%$; another variation on universal screening, where we assumed a socioeconomic differential in screening uptake, with the most deprived of the population to be $10 \%$ less likely to participate; and a variation on the population-wide intervention, where we only modelled dietary interventions, excluding smoking interventions. The supplementary file provides detailed information on the extra scenarios.

\section{Validation}

We assessed the predictive validity of the IMPACT $_{\mathrm{NCD}}$ model by comparing the estimated number of deaths from CVD with the observed number of deaths from the same causes for 2006 to 2013 in England. ${ }^{60}$ We further compared the IMPACT $_{\mathrm{NCD}}$ output with CVD mortality forecasts from a bayesian age-period-cohort model. ${ }^{61}$

\section{Patient involvement}

No patients were involved in setting the research question or the outcome measures, nor were they involved in developing plans for design or implementation of the study. No patients were asked to advise on interpretation or writing up of results. There are no plans to disseminate the results of the research to study participants or the relevant patient community.

\section{Results}

IMPACT $_{\mathrm{NCD}}$ outputs for CVD burden and inequality are summarised for ages 30 to 84 . Because of the assumed five year time lag, the interventions affect the population from 2016 up to the projection horizon of 2030. The impact of the five scenarios on risk factor trajectories are further illustrated in additional graphs in the supplementary file.

\begin{tabular}{lll}
\hline \multirow{2}{*}{ Table $1 \mid$ Estimated cases and deaths prevented or postponed under each scenario, by $\mathbf{2 0 3 0}$} \\
\cline { 2 - 3 } Scenarios & No (interquartile range) prevented or postponed \\
\cline { 2 - 3 } & Cases & Deaths \\
\hline Universal screening & $19000(11000-28000)$ & $3000(-1000-6000)$ \\
\hline Concentrated screening & $17000(9000-26000)$ & $8000(-1000-5000)$ \\
\hline Population-wide intervention & $67000(57000-77000)$ & $9000(6000-13000)$ \\
\hline $\begin{array}{l}\text { Population-wide intervention and } \\
\text { concentrated screening }\end{array}$ & $82000(73000-93000)$ & \\
\hline Results rounded to nearest 1000. & &
\end{tabular}

\section{Overall effectiveness}

Under the baseline scenario, IMPACT $\mathrm{NCD}_{\mathrm{N}}$ estimated about 1.4 million (interquartile range 1.3-1.5) cases of CVD and 540000 deaths (interquartile range 520000 to 550000 ) between 2016 and 2030. The most effective intervention was the combination of the population-wide intervention and concentrated screening. The population-wide intervention alone had the second highest effectiveness, whereas the universal and the concentrated screening scenarios were considerably less effective (table 1). Despite the improvement of most CVD related risk factors, the proportion of high risk people in the eligible population is slowly increasing over time, because of population aging (fig 1).

\section{Socioeconomic inequalities}

When socioeconomic inequalities were considered, the patterns for reductions in absolute and relative inequalities were similar. The combination of the population-wide intervention and concentrated screening seemed the most powerful among the simulated interventions (tables 2 and 3). Concentrated screening alone was the second most powerful intervention in tackling inequalities, followed by the population-wide intervention. Finally, universal screening of CVD is likely to have a small, if any, effect on socioeconomic inequalities.

\section{Equity summary chart}

We summarised our estimates for the effectiveness and equity of the modelled interventions in the equity summary chart (fig 2). The horizontal axis of the chart represents the cases of CVD prevented or postponed and the vertical axis the reduction in absolute inequality. Scenarios above the equity curve (dashed curve in the figure) decrease relative socioeconomic inequality, and scenarios below the curve increase it. The vertical distance from the curve approximates the impact of the scenario on relative inequality. (See the supplementary file for more details about this chart.) The combination of the population-wide intervention and concentrated screening is by far the most effective and equitable intervention. Concentrated screening is also equitable but with few mortality gains.

\section{Sensitivity analysis}

Adding assumptions to extend the scenarios did not displace our main findings. The three most notable results of the sensitivity analysis were:

Raising the treatment threshold from $10 \%$ to $20 \%$ further reduced the effectiveness of universal screening by about $60 \%$ in preventing CVD cases. However, in preventing deaths from CVD the effectiveness decreased by only $15 \%$ as raising the treatment threshold excludes younger participants at intermediate risk from treatment.

Assuming a differential uptake of universal screening by deprivation fifth essentially eliminated the estimated small potential benefit of universal screening in tackling health inequalities.

A population-wide intervention targeting only diet would still be about twice as effective as universal 


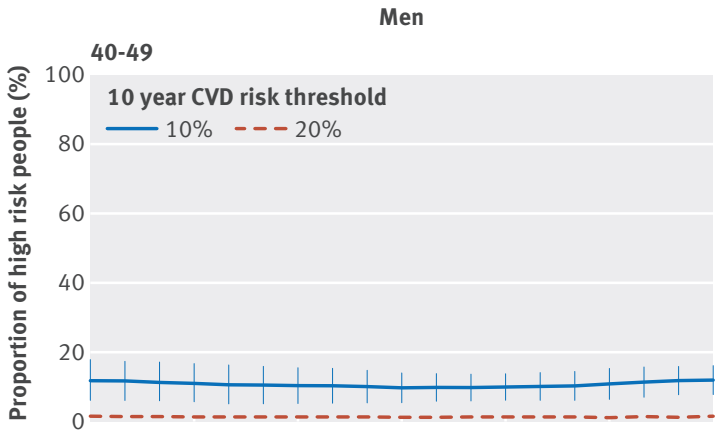

Women
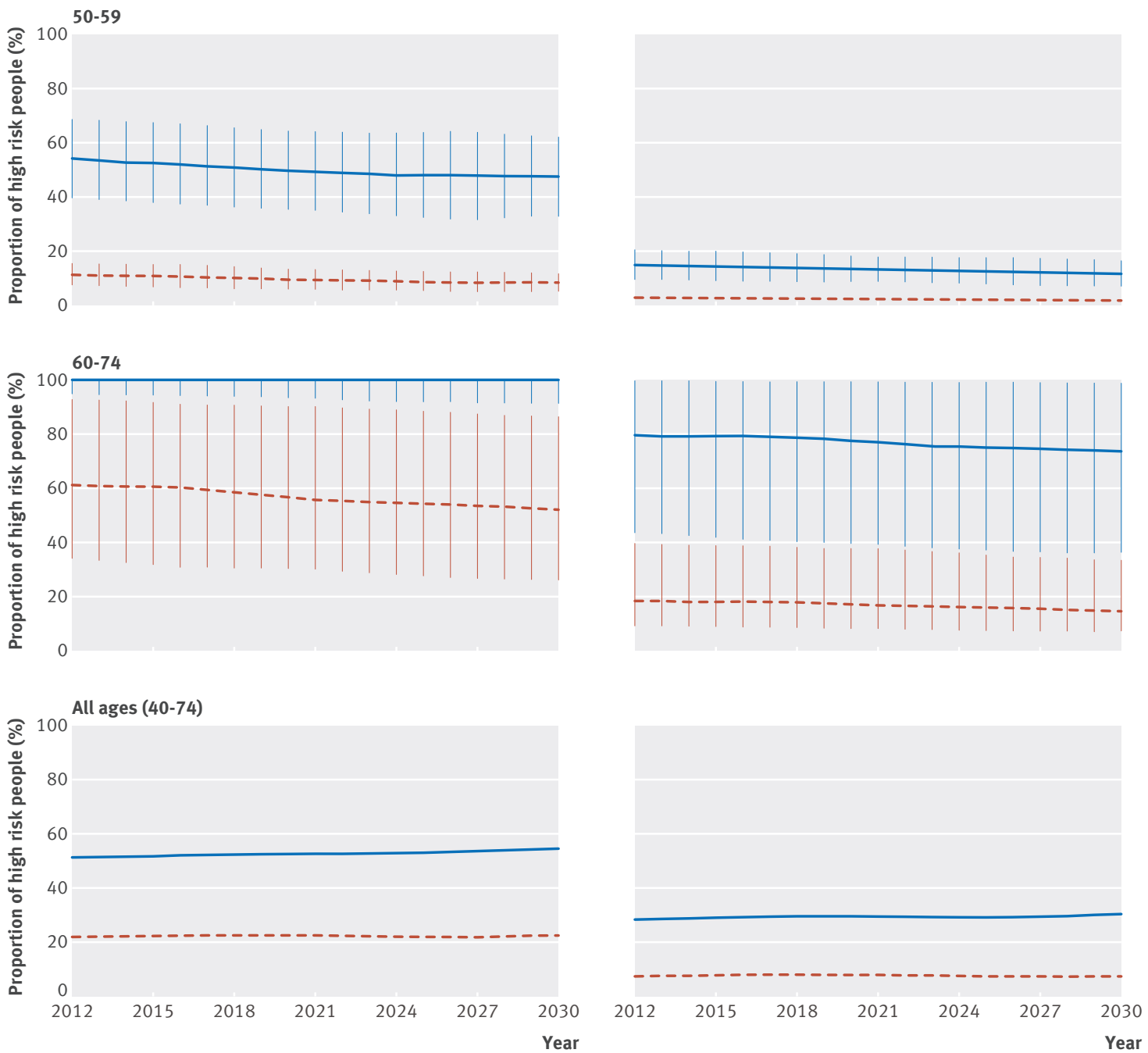

Fig 1 | Proportion of high risk people eligible for universal screening population projections, by age group and sex. 10 year risk of cardiovascular disease (CVD) was estimated from QRISK2 score. Error bars represent interquartile ranges

screening and more than twice as effective as population-wide intervention targeting smoking alone-so the relative ranking of scenario effectiveness would remain unaltered. For detailed results see supplementary tables S11-S13.

\section{Validation}

We assessed the predictive validity of the IMPACT $_{\mathrm{NCD}}$ model by comparing the estimated number of deaths from CVD with the observed number of deaths from the same cause for 2006 to 2013 in England (fig 3). See the supplementary file for detailed graphs by age group, sex, deprivation fifth, and disease.

\section{Discussion}

Our results strongly suggest that universal screening and treatment of people at high risk is not the most effective option for primary prevention of cardiovascular disease (CVD) overall, nor for reducing socioeconomic inequalities. In contrast, prevention 


\begin{tabular}{|c|c|c|c|c|}
\hline \multirow[b]{2}{*}{ Deprivation fifth* } & \multicolumn{4}{|c|}{ No (interquartile range) of cases prevented or postponed } \\
\hline & Universal screening & $\begin{array}{l}\text { Concentrated } \\
\text { screening }\end{array}$ & $\begin{array}{l}\text { Population-wide } \\
\text { intervention }\end{array}$ & $\begin{array}{l}\text { Population-wide intervention+ } \\
\text { concentrated screening }\end{array}$ \\
\hline First (least deprived) & $3400(-1400-8300)$ & 0 & $10800(5900-15500)$ & $10800(6200-15700)$ \\
\hline Second & $2900(-1500-8400)$ & 0 & $12200(6200-17200)$ & $11500(6600-17000)$ \\
\hline Third & $4000(-900-9300)$ & 0 & $13100(8100-18300)$ & $12600(7400-17700)$ \\
\hline Fourth & $3700(-1600-8600)$ & $6400(1500-11800)$ & $12500(7100-18400)$ & $18700(13900-24200)$ \\
\hline Fifth (most deprived) & $4900(-600-10400)$ & $10700(5300-16300)$ & $18700(13000-24000)$ & $28600(22800-33200)$ \\
\hline Absolute equity slope index & $1700(-6200-9300)$ & $14100(5700-23000)$ & $8400(-400-16900)$ & $21100(12800-29300)$ \\
\hline
\end{tabular}

\begin{tabular}{|c|c|c|c|c|}
\hline \multirow[b]{2}{*}{ Deprivation fifth* } & \multicolumn{4}{|c|}{ Relative $\%$ reduction (interquartile range) } \\
\hline & Universal screening & $\begin{array}{l}\text { Concentrated } \\
\text { screening }\end{array}$ & $\begin{array}{l}\text { Population-wide } \\
\text { intervention }\end{array}$ & $\begin{array}{l}\text { Population-wide intervention+ } \\
\text { concentrated screening }\end{array}$ \\
\hline First (least deprived) & $1.3(-0.5-3.1)$ & 0 & $4.1(2.2-5.9)$ & $4.0(2.4-6.0)$ \\
\hline Second & $1.1(-0.5-2.9)$ & 0 & $4.2(2.2-5.9)$ & $4.0(2.3-5.9)$ \\
\hline Third & $1.4(-0.3-3.2)$ & 0 & $4.6(2.8-6.3)$ & $4.4(2.6-6.2)$ \\
\hline Fourth & $1.3(-0.6-3.1)$ & $2.4(0.6-4.3)$ & $4.6(2.7-6.6)$ & $6.9(5.1-8.9)$ \\
\hline Fifth (most deprived) & $1.6(-0.2-3.3)$ & $3.6(1.8-5.3)$ & $6.2(4.4-8.0)$ & $9.4(7.6-11.2)$ \\
\hline Relative equity slope index & $0.4(-2.4-3.2)$ & $4.9(1.8-7.9)$ & $2.3(-0.7-5.3)$ & $6.7(3.8-9.5)$ \\
\hline
\end{tabular}

strategies that include population-wide structural interventions seem to be the consistently better options for reducing overall CVD burden and inequalities. This echoes and quantifies findings from other, mostly theoretical, studies supporting that structural population-wide interventions are powerful, while reducing socioeconomic health inequalities. ${ }^{13} 146263$ Indeed, the impact of the population-wide intervention scenario on reduction in estimated mortality and inequalities seems compatible with previous estimates, considering the different methodologies. ${ }^{64}$ Furthermore, the effectiveness and equity of population-wide structural interventions can be further improved by the addition of targeted interventions in the most deprived groups, as highlighted in the combined scenario of the population-wide intervention and concentrated screening.

Compared with other modelling approaches, our IMPACT $_{\mathrm{NCD}}$ model estimated that NHS Health Checks might prevent approximately 1000 non-fatal and 200 fatal cases of CVD annually. This is comparable with the Department of Health estimates of 1600 non-fatal CVD cases and 650 deaths prevented annually. ${ }^{4}$ Furthermore, the Department of Health modelling approach assumed an intervention uptake of $75 \%$; higher than the current observed levels. Using the Archimedes model, Schuetz et al estimated that health checks in the UK could prevent some 12 CVD cases per 1000 population screened after 30 years' follow-up ${ }^{65}$ (7500 CVD cases prevented each year extrapolating to the eligible English population). That higher estimate reflects the researchers' apparently unrealistic assumption of $100 \%$ screening uptake and $50 \%$ overall uptake of treatment.

\section{The scenarios}

We modelled the universal screening scenario to closely resemble the current implementation of the NHS Health Check programme, based on published evidence. Therefore, we maintain that our estimates on the effectiveness of this scenario are not far from the real world effectiveness of NHS Health Checks. However, our output suggesting that universal screening might reduce socioeconomic inequalities seems to contradict existing empirical and modelling evidence. ${ }^{1419-21}$ This is because we generously assumed identical screening uptake and treatment adherence for all socioeconomic groups. In fact, any potential reduction in socioeconomic health inequalities was essentially eliminated when we considered a small socioeconomic differential in uptake in the sensitivity analysis. Furthermore, additional health inequalities may arise from differential persistence and adherence to treatment by deprivation status. ${ }^{46}$

The population-wide intervention scenario on the other hand, is based mostly on structural policies targeting price and availability. This scenario potential effectiveness was mostly based on natural experiments, ${ }^{6667}$ and on previous modelling studies from the UK and elsewhere. The size of the changes in the population risk factors that we modelled were modest, and actually smaller than the reductions observed in countries such as France, Finland, and the USA during recent decades. ${ }^{68-70}$ This scenario estimated the reduction in mortality conservatively, because it ignored the beneficial effect of the policies on survival from CVD. Similarly, it underestimated the reduction of the gap in inequalities, because it did not fully consider the current disproportionate burden of poor diet among the 


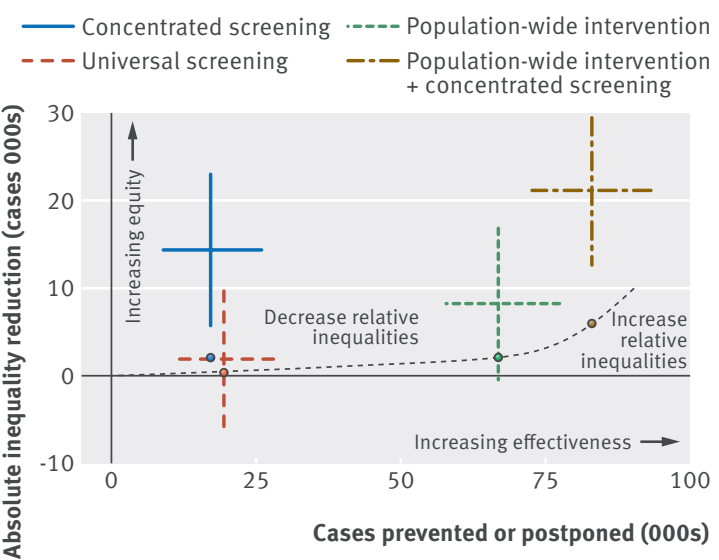

Fig 2 | Equity summary chart of effectiveness and equity of all modelled interventions, compared with baseline scenario (beginning of axes). Dashed line represents "equity" curve. Interventions below the curve increase relative inequality, whereas interventions above it decrease relative inequalities. Smaller coloured dots represent reference points used to fit equity curve. Horizontal and vertical error bars represent interquartile ranges

most deprived of the population, ${ }^{71}$ and hence the potential for improvement through population-wide policies.

Finally, the concentrated screening strategy was the weakest in terms of overall effectiveness, yet more powerful in tackling inequalities. Its increased impact on socioeconomic health inequalities is a direct consequence of the concentrated prevention only to the more
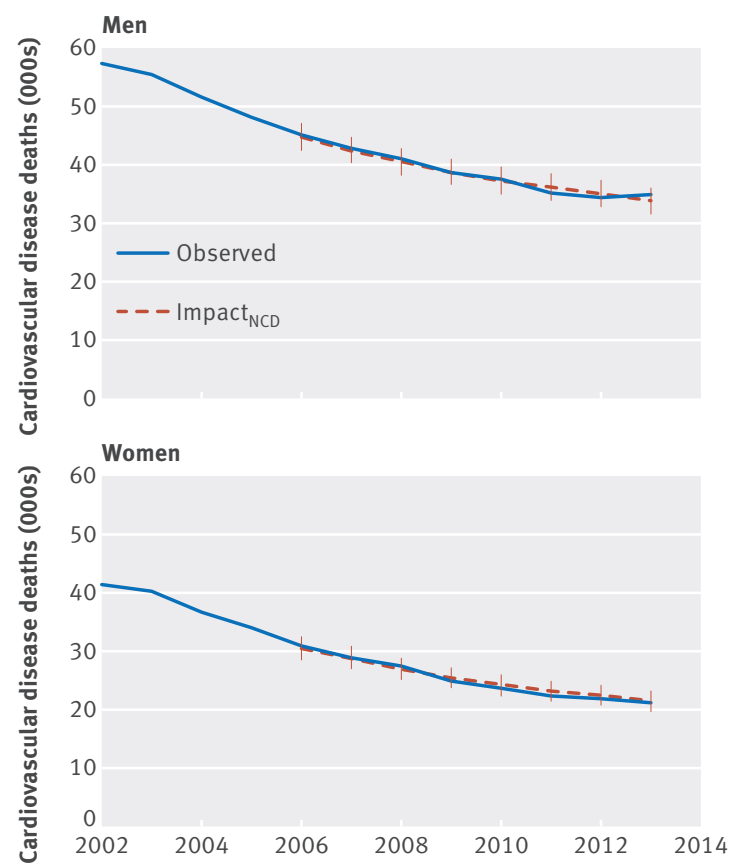

Year

Fig 3 | Number of deaths from cardiovascular disease (CVD) in England, by year for ages 30 to 84 . Office for National Statistics reported deaths (observed) versus IMPACT ${ }_{N C D}$ estimated. Observed deaths after 2010 were adjusted to account for changes in ICD-10 version used by the Office for National Statistics from 2011 onwards. Error bars represent interquartile ranges deprived quantiles of the population. However, the scenario assumptions may not fully hold in real world implementation. Hence, concentrated screening represents a challenge for public health practitioners and policymakers to exploit the opportunity of a smaller and more homogeneous eligible population and to implement better recruitment and tactics for treatment adherence. Yet, cost effectiveness might also fall because of loss of economies of scale.

\section{Public health implications}

This IMPACT $_{\mathrm{NCD}}$ modelling may help stakeholders to understand better the interplay between preventive policies, risk factors, disease, and inequalities, and thus potentially inform health policy and strategy. Hence, when compared with the alternative feasible interventions, universal screening seemed inferior both in primary prevention and in reducing socioeconomic health inequalities. Additionally, we estimated that the proportion of young people at high risk aged less than 60 in the eligible population will decrease in future (fig 1). This will render universal screening less effective and less cost effective for this age group, because a larger number will need to be screened to identify each high risk individual.

Our study suggests that despite the high clustering of risk factors in the most deprived parts of the population, structural population-wide approaches remain more effective than high risk ones for the prevention of CVD. Population-wide approaches also seem to be more effective in reducing absolute and relative socioeconomic health inequalities, generally cost much less than a universal screening programme, and may even be cost saving. ${ }^{7273}$ In this study, we did not model the full potential of these policies, as we focused only on diet and smoking interventions; we did not, for example, incorporate alcohol consumption or physical activity. In addition, we did not simulate the likely wider benefits of improved diet and smoking cessation on the plethora of relevant non-communicable diseases. Despite this restricted scope, for CVD prevention we estimated that structural policies targeting diet could be twice as effective as those targeting smoking. Yet, structural interventions for a healthier diet are currently underutilised compared with tobacco control. Several countries have now introduced taxes on sugary drinks or sugar, including Finland, France, Latvia, and Mexico. The UK has recently followed their example. Hungary is the only European country currently taxing unhealthy "junk" food. ${ }^{74}$ However, fiscal interventions may face opposition from commercial vested interests. ${ }^{75}$ Interestingly, an increasing body of evidence from empirical studies and modelling analyses suggest that the maximum health impact with a neutral effect on poverty may occur when food or drinks taxes are combined with subsidies for healthy foods. ${ }^{567677}$

Moreover, the combination of a population-wide intervention with an intervention targeting the most deprived members, may further improve effectiveness and equity. This approach is in the spirit of proportionate universalism that was identified in the Marmot 
review as the best approach to tackle socioeconomic inequalities in health. ${ }^{78}$ Our study provides evidence that in CVD prevention proportionate universalism may be the best option not only for tackling inequalities but also for overall effectiveness.

\section{Strengths and limitations of this study}

IMPACT $_{\mathrm{NCD}}$ is the first microsimulation model to synthesise core principles of social and CVD epidemiology, vital demographics, published literature, and recent health surveys for England to create a synthetic population of England, including socioeconomic structure, at the individual level. The microsimulation approach allows for the simulation of detailed scenarios and explores the distributional nature of their impact on the population, in a competing risks framework. Microsimulation allows for greater flexibility and more detailed simulation, demanding more statistical and computational resources than older approaches; we utilised the Farr Institute's statistical high performance computing facilities. ${ }^{79}$ Many assumptions must be made with such models. Yet, despite the potential frailty of such assumptions, this model validated well against observed CVD mortality, even when multiply stratified. Finally, to ensure transparency, we have made the IMPACT $_{\mathrm{NCD}}$ source code open under GNU GPLv3 license.

Models are simplifications of reality and thus possess inherent limitations. At least four items were not included in the current model. Firstly, the multiplicative risk assumption is considered the status quo in comparative risk assessments; ${ }^{80}$ however, this may oversimplify the complex nature of interactions between multiple risk factors and disease outcome over the life course. Secondly, IMPACT $_{\mathrm{NCD}}$ currently ignores the effect of risk factors on CVD case fatality, although in this study we considered only primary prevention scenarios. Thirdly, complex population dynamics such as migration, social mobility, and the socioeconomic consequences of disease were not modelled. We consider this bias would be relatively small for projections with a short horizon. Fourthly, the model ignores the impact of universal screening in recognising previously undiagnosed cases of atrial fibrillation and other opportunistic diagnoses. Reassuringly, most of these biases apply across all scenarios; their effects would thus be reduced in comparisons between scenarios.

\section{Conclusions}

When comparing primary prevention strategies for reducing CVD burden and inequalities, universal screening seems less effective than alternative strategies that incorporate population-wide approaches. Further research is needed to identify the best mix of population-wide and risk targeted CVD strategies to maximise cost effectiveness and minimise inequalities.

We thank Julia Critchley for her valuable advice about modelling physical activity, and the reviewers and editorial committee for their comments and suggestions, which have greatly improved the original manuscript.

Contributors: All authors made substantial contributions to the conception and design of the study. CK and MOF had the original idea.
CK prepared and conducted data analysis. All authors drafted and critically revised the manuscript. All authors, external and internal, had full access to all of the data (including statistical reports and tables) in the study and can take responsibility for the integrity of the data and the accuracy of the data analysis. CK is the guarantor of this study.

Funding: The health survey for England was funded by the Department of Health until 2004 and the Health and Social Care Information Centre from 2005. IB and CK were supported by Medical Research Council Health eResearch Centre grant MR/K006665/1. SC, MOF, MGC, KA, and PB were supported by the National Institute for Health Research through a grant (SPHR-LIL-PH1-MCD) to the LiLaC collaboration between the University of Liverpool and Lancaster University. The funders had no role in the study design, data collection and analysis, decision to publish, or preparation of the manuscript

Competing interests: All authors have completed the ICMJE uniform disclosure form at http://www.icmje.org/coi_disclosure.pdf and declare: no support from any organisation for the submitted work; no financial relationships with any organisations that might have an interest in the submitted work in the previous three years, no other relationships or activities that could appear to have influenced the submitted work

Ethical approval: Not required as this study is an analysis of previously collected data. Ethical approval for each survey was obtained by the health survey for England team.

Data sharing: Anonymised, non-identifiable participant level cross sectional survey data are freely available for academic researchers and public health staff to download from the UK data service (www. ukdataservice.ac.uk). The source code for IMPACT ISCD $_{\text {is available at }}$ https://github.com/christk/impactncd/tree/cvd-policy-options.

Transparency: The lead author (the manuscript's guarantor) affirms that this manuscript is an honest, accurate, and transparent account of the study being reported; that no important aspects of the study have been omitted; and that any discrepancies from the study as planned have been explained.

This is an Open Access article distributed in accordance with the terms of the Creative Commons Attribution (CC BY 3.0) license, which permits others to distribute, remix, adapt and build upon this work, for commercial use, provided the original work is properly cited. See: http://creativecommons.org/licenses/by/3.0/

1 GBD 2013 Mortality and Causes of Death Collaborators. Global, regional, and national age-sex specific all-cause and causespecific mortality for 240 causes of death, 1990-2013: a systematic analysis for the Global Burden of Disease Study 2013. Lancet 2015;385:117-71. doi:10.1016/S0140-6736(14)61682-2.

2 Townsend N, Wickramasinghe K, Bhatnagar P, et al. Coronary heart disease statistics 2012 edition. British Heart Foundation 2012 . www. bhf.org.uk/publications/view-publication.aspx?ps=1002097.

3 Mackenbach JP, Cavelaars AE, Kunst AE, Groenhof F. Socioeconomic inequalities in cardiovascular disease mortality; an international study. Eur Heart J 2000;21:1141-51. doi:10.1053/euhj.1999.1990.

4 Department of Health. Cardiovascular disease outcomes strategy: improving outcomes for people with or at risk of cardiovascular disease. 2013. www.gov.uk/government/uploads/system/uploads/ attachment_data/file/217118/9387-2900853-CVD-Outcomes_ web1.pdf.

Choices NHS. NHS Health Check www healthcheck nhs.uk/.

6 Capewell S, McCartney M, Holland W. Invited debate: NHS Health Checks-a naked emperor? J Public Health 2015;37:187-92. doi:10.1093/pubmed/fdv063.

7 Waterall J, Greaves F, Kearney M, et al. Invited debate: NHS Health Check: an innovative component of local adult health improvement and well-being programmes in England. J Public Health 2015;37:17784. doi:101093/pubmed/fdv062.

8 Braillon A, Bewley S, Pisinger C, Fisken RA, Richmond C. NHS health checks are a waste of resources. BMJ 2015;350:h1006. doi:10.1136/ bmi.h1006.

9 O'Dowd A. MPs call for review of NHS health checks. BMJ 2014;349:g6498-6498. doi:10.1136/bmj.g6498.

10 Department of Health. Government response to the House of Commons Science and Technology Committee report on national health screening. 2015. www.gov.uk/government/publications/ national-health-screening-government-response.

11 He FI, Brinsden HC, MacGregor GA. Salt reduction in the United Kingdom: a successful experiment in public health. J Hum Hypertens 2014;28:345-52. doi:10.1038/jhh.2013.105

12 Levy DT, Currie L, Clancy L. Tobacco control policy in the UK: blueprint for the rest of Europe? Eur I Public Health 2013:23:201-6. doi:10.1093/eurpub/cks090.

13 Rose G. Sick individuals and sick populations. Int J Epidemiol 2001;30:427-32, discussion 433-4. doi:10.1093/ije/30.3.427. 
14 Capewell S, Graham H. Will cardiovascular disease prevention widen health inequalities? PLoS Med 2010;7:e1000320. doi:10.1371/ journal.pmed.1000320.

15 Krogsbøll LT, Jørgensen KJ, Grønhøj Larsen C, Gøtzsche PC. General health checks in adults for reducing morbidity and mortality from disease: Cochrane systematic review and meta-analysis. BMJ 2012;345:e7191. doi:10.1136/bmj.e7191.

16 Jørgensen T, Jacobsen RK, Toft U, Aadahl M, Glümer C, Pisinger C. Effect of screening and lifestyle counselling on incidence of ischaemic heart disease in general population: Inter99 randomised trial. BMJ 2014;348:g3617-3617. doi:10.1136/bmj.g3617.

17 Manuel DG, Lim J, Tanuseputro P, et al. Revisiting Rose: strategies for reducing coronary heart disease. BM/ 2006;332:659-62. doi:10.1136/ bmj.332.7542.659.

18 Scholes S, Bajekal M, Love H, et al. Persistent socioeconomic inequalities in cardiovascular risk factors in England over 1994-2008: a time-trend analysis of repeated cross-sectional data. BMC Public Health 2012;12:129. doi:10.1186/1471-2458-12-129.

19 White M, Adams J, Heywood P. How and why do interventions that increase health overall widen inequalities within populations? In: Babones SI, ed. Social inequality and public health. Policy Press, 2009: 64-81, http://policypress.universitypressscholarship.com/view/10.1332/ policypress/9781847423207.001.0001/upso-9781847423207-chapter5doi:10.1332/policypress/9781847423207.003.0005.

20 Wallach-Kildemoes H, Diderichsen F, Krasnik A, Lange T, Andersen M. Is the high-risk strategy to prevent cardiovascular disease equitable? A pharmacoepidemiological cohort study. BMC Public Health 2012;12:610. doi:10.1186/1471-2458-12-610.

21 Bender AM, Kawachi I, Jørgensen T, Pisinger C. Neighborhood deprivation is strongly associated with participation in a populationbased health check. PLoS One 2015;10:e0129819. doi:10.1371/ journal.pone.0129819.

22 Chang KC-M, Soljak M, Lee JT, et al. Coverage of a national cardiovascular risk assessment and management programme (NHS Health Check): Retrospective database study. Prev Med 2015;78:1-8 doi:10.1016/j.ypmed.2015.05.022.

23 Gidlow C, Ellis N, Randall J, et al. Method of invitation and geographical proximity as predictors of NHS Health Check uptake. J Public Health 2014;26. doi:10.1093/pubmed/fdu092.

24 Attwood S, Morton K, Sutton S. Exploring equity in uptake of the NHS Health Check and a nested physical activity intervention trial. J Public Health (Oxf) 2015;01:fdv070. doi:10.1093/pubmed/fdv070.

25 Baker C, Loughren EA, Crone D, Kallfa N. A process evaluation of the NHS Health Check care pathway in a primary care setting. I Public Health (Oxf) 2015;37:202-9. doi:10.1093/pubmed/fdv053.

26 Unal B, Critchley JA, Capewell S. Explaining the decline in coronary heart disease mortality in England and Wales between 1981 and 2000. Circulation 2004:109:1101-7. doi:10.1161/01. CIR.0000118498.35499.B2

27 Bajekal M, Scholes S, Love H, et al. Analysing recent socioeconomic trends in coronary heart disease mortality in England, 2000-2007: a population modelling study. PLoS Med 2012;9:e1001237. doi:10.1371/journal.pmed.1001237.

28 Ainsworth JD, Carruthers E, Couch P, et al. IMPACT: a generic tool for modelling and simulating public health policy. Methods InfMed 2011;50:454-63. doi:10.3414/ME11-02-0006

29 Department for Communities and Local Government. English indices of deprivation 2010 - Publications - GOV.UK. 2011. www.gov.uk/ government/statistics/english-indices-of-deprivation-2010.

30 Mindell J, Biddulph JP, Hirani V, et al. Cohort profile: the health survey for England. Int J Epidemiol 2012;41:1585-93. doi:10.1093/ ije/dyr199.

31 National Centre for Social Research, University College London. Department of Epidemiology and Public Health. Health Survey for England, 2001. [computer file] 3rd ed. UK Data Archive, 2010. [distributor], 10.5255/UKDA-SN-4628-1.

32 National Centre for Social Research, University College London. Department of Epidemiology and Public Health. Health Survey for England, 2002. [computer file] 2nd ed. UK Data Archive, 2010. [distributor], 10.5255/UKDA-SN-4912-1.

33 National Centre for Social Research, University College London. Department of Epidemiology and Public Health. Health Survey for England, 2003. [computer file] 2nd ed. UK Data Archive, 2010. [distributor], 10.5255/UKDA-SN-5098-1.

34 National Centre for Social Research, University College London. Department of Epidemiology and Public Health. Health Survey for England, 2004. [computer file] 2nd ed. UK Data Archive, 2010. [distributor], 10.5255/UKDA-SN-5439-1.

35 National Centre for Social Research, University College London. Department of Epidemiology and Public Health. Health Survey for England, 2005. [computer file] 3rd ed. UK Data Archive, 2011. [distributor], 10.5255/UKDA-SN-5675-1.

36 National Centre for Social Research, University College London. Department of Epidemiology and Public Health. Health Survey for England, 2006. [computer file] 4th ed. UK Data Archive, 2011. [distributor], 10.5255/UKDA-SN-5809-1.
37 National Centre for Social Research, University College London. Department of Epidemiology and Public Health. Health Survey for England, 2007. [computer file] 2nd ed. UK Data Archive, 2010. [distributor], 10.5255/UKDA-SN-6112-1.

38 National Centre for Social Research, University College London. Department of Epidemiology and Public Health. Health Survey for England, 2008. [computer file] 4th ed. UK Data Archive, 2013. [distributor], 10.5255/UKDA-SN-6397-2.

39 National Centre for Social Research, University College London. Department of Epidemiology and Public Health. Health Survey for England, 2009. [computer file] 2nd ed. UK Data Archive, 2011. [distributor], 10.5255/UKDA-SN-6732-1.

40 NatCen Social Research, Free Royal, University College London. Department of Epidemiology and Public Health. Health Survey for England, 2010. [computer file] 2nd ed. UK Data Archive, 2012. [distributor], 10.5255/UKDA-SN-6986-2.

41 NatCen Social Research, University College London. Department of Epidemiology and Public Health. Health Survey for England, 2011. [computer file] UK Data Archive, 2013. [distributor], 10.5255/ UKDA-SN-7260-1.

42 NatCen Social Research, University College London. Department of Epidemiology and Public Health. Health Survey for England, 2012. [computer file] UK Data Archive, 2014. [distributor], 10.5255/ UKDA-SN-7480-1.

43 Public Health England. Explore NHS Health Check Data. www. healthcheck.nhs.uk/interactive_map/.

44 Collins GS, Altman DG. Predicting the 10 year risk of cardiovascular disease in the United Kingdom: independent and external validation of an updated version of QRISK2. BMJ 2012;344:e4181. doi:10.1136/ bmi.e4181.

45 Forster AS, Dodhia $\mathrm{H}$, Booth $\mathrm{H}$, et al. Estimating the yield of NHS Health Checks in England: a population-based cohort study. J Public Health (Oxf) 2015;37:234-40. doi:10.1093/pubmed/fdu079.

46 Wallach-Kildemoes H, Andersen M. Diderichsen F, Lange T. Adherence to preventive statin therapy according to socioeconomic position. Eur J Clin Pharmacol 2013;69:1553-63. doi:10.1007/ s00228-013-1488-6.

47 Hutfless S, Gudzune KA, Maruthur N, et al. Strategies to prevent weight gain in adults: a systematic review. Am J Prev Med 2013;45:e41-51. doi:10.1016/j.amepre.2013.07.013.

48 Bauld L, Bell K, McCullough L, Richardson L, Greaves L. The effectiveness of NHS smoking cessation services: a systematic review. J Public Health (Oxf) 2010;32:71-82. doi:10.1093/pubmed/ $\mathrm{fdp} 074$

49 West R. Lesley Owen. Estimates of 52-week continuous abstinence rates following selected smoking cessation interventions in England. Smoking in England 2012. www.smokinginengland.info/ downloadfile/?type $=$ report\&src $=5$.

50 Sharma A, Hauck K, Hollingsworth B, Siciliani L. The effects of taxing sugar-sweetened beverages across different income groups. Health Econ 2014;23:1159-84. doi:10.1002/hec.3070.

51 Briggs ADM, Mytton OT, Kehlbacher A, Tiffin R, Rayner M, Scarborough P. Overall and income specific effect on prevalence of overweight and obesity of $20 \%$ sugar sweetened drink tax in UK: econometric and comparative risk assessment modelling study. BMJ 2013:347:f6189. doi:10.1136/bmj.f6189.

52 Cabrera Escobar MA, Veerman JL, Tollman SM, Bertram MY, Hofman KJ. Evidence that a tax on sugar sweetened beverages reduces the obesity rate: a meta-analysis. BMC Public Health 2013;13:1072. doi:10.1186/1471-2458-13-1072.

53 Webster LL, Dunford EK, Hawkes C, Neal BC. Salt reduction initiatives around the world. J Hypertens 2011;29:1043-50. doi:10.1097/ HJH.Ob013e328345ed83.

54 Gillespie DOS, Allen K, Guzman-Castillo M, et al. The health equity and effectiveness of policy options to reduce dietary salt intake in England: policy forecast. PLoS One 2015;10:e0127927. doi:10.1371/ journal.pone.0127927.

55 Bartlett S, Klerman J, Olsha L, et al. Evaluation of the Healthy Incentives Pilot (HIP) Final Report. 2014. www.fns.usda.gov/sites/ default/files/HIP-Final.pdf.

56 Nnoaham KE, Sacks G, Rayner M, Mytton O, Gray A. Modelling income group differences in the health and economic impacts of targeted food taxes and subsidies. Int J Epidemiol 2009;38:1324-33. doi:10.1093/ije/dyp214.

57 Levy DT, Huang A-T, Currie LM, Clancy L. The benefits from complying with the framework convention on tobacco control: a SimSmoke analysis of 15 European nations. Health Policy Plan 2014;29:1031-42. doi:10.1093/heapol/czt085.

58 Alfons A, Kraft S, Templ M, et al. Simulation of close-to-reality population data for household surveys with application to EU-SILC. Stat Methods Appl 2011;20:383-407. doi:10.1007/ s10260-011-0163-2.

59 Mackenbach JP, Kunst AE. Measuring the magnitude of socioeconomic inequalities in health: an overview of available measures illustrated with two examples from Europe. Soc Sci Med 1997;44:75771. doi:10.1016/S0277-9536(96)00073-1. 
60 Office for National Statistics. Results of the ICD-10 v2010 bridge coding study, England and Wales. 2011. www.ons.gov.uk/ons/rel/ subnational-health3/results-of-the-icd-10-v2010-bridge-codingstudy--england-and-wales--2009/2009/index.html.

61 Schmid VJ, Held L. Bayesian age-period-cohort modeling and prediction-BAMP. J Stat Softw 2007;21:1-15doi:10.18637/jss.v021.i08.

62 Lorenc T, Petticrew M, Welch V, Tugwell P. What types of interventions generate inequalities? Evidence from systematic reviews. J Epidemio Community Health 2013;67:190-3. doi:10.1136/jech-2012-201257.

63 McLaren L, McIntyre L, Kirkpatrick S. Rose's population strategy of prevention need not increase social inequalities in health. Int J Epidemiol 2010;39:372-7. doi:10.1093/ije/dyp315.

64 Scholes S, Bajekal M, Norman P, et al. Quantifying policy options for reducing future coronary heart disease mortality in England: a modelling study. PLoS One 2013;8:e69935. doi:10.1371/journal. pone.0069935.

65 Schuetz CA, Alperin P, Guda S, et al. A standardized vascular disease health check in europe: a cost-effectiveness analysis. PLoS One 2013;8:e66454. doi:10.1371/journal.pone.0066454

66 Bandosz P, O'Flaherty M, Drygas W, et al. Decline in mortality from coronary heart disease in Poland after socioeconomic transformation: modelling study. BMJ 2012;344:d8136. doi:10.1136/bmj.d8136.

67 Franco M, Bilal U, Orduñez P, et al. Population-wide weight loss and regain in relation to diabetes burden and cardiovascular mortality in Cuba 1980-2010: repeated cross sectional surveys and ecological comparison of secular trends. BMJ 2013;346:f1515. doi:10.1136/bmj. f1515.

68 Danaei G, Finucane MM, Lin JK, et al. Global Burden of Metabolic Risk Factors of Chronic Diseases Collaborating Group (Blood Pressure). National, regional, and global trends in systolic blood pressure since 1980: systematic analysis of health examination surveys and epidemiological studies with 786 country-years and 5.4 million participants. Lancet 2011;377:568-77. doi:10.1016/ S0140-6736(10)62036-3.

69 Farzadfar F, Finucane MM, Danaei G, et al. Global Burden of Metabolic Risk Factors of Chronic Diseases Collaborating Group (Cholesterol). National, regional, and global trends in serum total cholesterol since 1980: systematic analysis of health examination surveys and epidemiological studies with 321 country-years and 3.0 million participants. Lancet 2011;377:578-86. doi:10.1016/ S0140-6736(10)62038-7.
70 Molarius A, Parsons RW, Dobson AJ, et al. WHO MONICA Project. Trends in cigarette smoking in 36 populations from the early 1980 s to the mid-1990s: findings from the WHO MONICA Project. Am I Public Health 2001;91:206-12. doi:10.2105/AJPH.91.2.206

71 Maguire ER, Monsivais P. Socio-economic dietary inequalities in UK adults: an updated picture of key food groups and nutrients from national surveillance data. Br J Nutr 2015;113:181-9. doi:10.1017/ S0007114514002621.

72 Barton P, Andronis L, Briggs A, McPherson K, Capewell S. Effectiveness and cost effectiveness of cardiovascular disease prevention in whole populations: modelling study. BMJ 2011;343:d4044. doi:10.1136/ bmj.d4044.

73 Vos T, Carter R, Barendregt J, et al. Assessing Cost-Effectiveness in Prevention. The University of Queensland, Brisbane, and Deakin University, 2010, www.sph.uq.edu.au/docs/BODCE/ACE-P/ ACE-Prevention_final_report.pdf.

74 Lloyd-Williams F, Bromley H, Orton L, et al. Smorgasbord or symphony? Assessing public health nutrition policies across 30 European countries using a novel framework. BMC Public Health 2014:14:1195. doi:10.1186/1471-2458-14-1195.

75 Bødker M, Pisinger C, Toft U, Jørgensen T. The rise and fall of the world's first fat tax. Health Policy 2015;119:737-42. doi:10.1016/j. healthpol.2015.03.003.

76 Leifert RM, Lucinda CR. Linear Symmetric 'Fat Taxes': Evidence from Brazil. Appl Econ Perspect Policy 2015;37:634-66. doi:10.1093/aepp/ ppu062.

77 Madden D. The poverty effects of a 'fat-tax' in Ireland. Health Econ 2015;24:104-21. doi:10.1002/hec.3006.

78 The Marmot Review. Fair society, healthy lives: strategic review of health inequalities in England post 2010.UCL Institute of Health Equity, 2010.

79 Farr Institute. Investment in supercomputer accelerates the pace of health research across the North of England. www.farrinstitute.org/news/ investment-in-supercomputer-accelerates-the-pace-of-health-researchacross-the-north-of-england.

80 World Health Organization. Comparative quantification of health risks.

WHO, 2004. www.who.int/healthinfo/global_burden_disease/cra/en/.

Appendix: supplementary information 